\title{
PERFIL SOCIODEMOGRÁFICO E QUALIDADE DE VIDA DE IDOSOS DE UM CENTRO DE REFERÊNCIA DO IDOSO DO OESTE PAULISTA
}

Isabela Cristina Duarte Araújo, Gislaine da Silva Toretto, Thaoane Aparecida Cano Toledo, Margarete Jardinetti de Oliveira, Aline Duarte Ferreira, Weber Gutemberg Alves de Oliveira

Universidade do Oeste Paulista - UNOESTE, Cursos de Fisioterapia e Medicina, Presidente Prudente/SP. E-mail: isabela_duarte@live.com

\section{RESUMO}

O objetivo deste estudo foi descrever o perfil sóciodemográfico, qualidade de vida e nível de atividade física de idosos do Centro de Referência do Idoso (CRI) comparados aos idosos do Brasil por plataforma governamental. Estudo comparativo e transversal, incluindo 100 idosos de ambos os gêneros com idade de $73,2 \pm 6$ anos. A qualidade de vida foi avaliada pelo questionário WHOQOL-OLD, o nível de atividade física pelo questionário Baecke e o nível cognitivo através do Mini Exame do Estado Mental. A análise intergrupos foi realizada pelo teste Qui-quadrado. Para análise do nível de atividade física utilizou-se o teste KruskalWallis $(p<0,05)$. Os resultados sociodemográficos mostraram que escolaridade, estado civil, renda e profissão foram superiores quando comparados aos dados nacionais e a ausência de dados da população idosa brasileira sobre nível de atividade física dificultou realizar comparações. Concluiu-se que a amostra encontra-se em nível igual ou superior na maioria dos perfis da população brasileira.

Palavras chave: envelhecimento, qualidade de vida, exercício, serviços de saúde para idosos, assistência a idosos.

\section{SOCIODEMOGRAPHIC PROFILE AND QUALITY OF LIFE OF ELDERLY IN REFERENCE CENTER IN THE WEST OF SÃO PAULO}

\begin{abstract}
\section{INTRODUÇÃO}

Desde 1950 o Brasil vem passando por um processo de transição demográfica, com uma redução da proporção da população jovem, e uma elevação da população adulta e principalmente idosa, isso se deve a alta longevidade e a baixa natalidade, representadas por queda dos níveis de mortalidade, fecundidade e natalidade ${ }^{1}$.
\end{abstract}

The study that was describing the profile of the socioeducation, quality of life, level of physical activities of the center of Elderly (CRI) and comparatives of the Brazilian plan by the governmental. A comparative and cross-sectional study involving 100 elderly women of both sexes, aged $73.2 \pm 6$ years. To assess quality of life, use the WHOQOL-OLD questionnaire, the level of physical activity through the questionnaire, and the cognitive level of the Mental State Mini Exam. The chi-square test was used to analyze the intergroups. The analysis of the level of physical activity was used as Kruskal-Wallis test $(p<0.05)$. Data on the sociodemographic aspects are related to schooling, marital status, income and child penetration, when compared to data from the Brazilian population, of Systemic Arterial Hypertension, similar to data such as morbidity and data from the elderly population on the level of physical activity made it difficult to compare. Essay is a population study found to be equal and superior in most profiles of the Brazilian population.

Keywords: aging, quality of life, exercise, health services for the aged, old age assistance.

O envelhecimento é responsável por muitas modificações fisiológicas e funcionais em cada indivíduo assim desencadeando o surgimento de doenças crônico-degenerativas. Fatores como estresse e sedentarismo são importantes por acelerar esse processo, trazendo sérios riscos para a saúde da população dos idosos $^{2}$. No atual contexto demográfico brasileiro, estas doenças crônicas ganham grande importância, pois acometem a maior parte da 
população idosa e contribuem para o aumento de cuidados e gastos em saúde ${ }^{3}$.

O trabalho multiprofissional faz-se importante como estratégias que incentivem os idosos a buscar uma melhora da sua saúde, com o intuito de ter uma qualidade de vida, melhorando consequentemente a sua autoestima no processo do envelhecimento ${ }^{4}$.

A qualidade de vida dos idosos tem sido um importante questionamento na sociedade moderna, segundo Deponti e Acosta ${ }^{5}$, para um melhor entendimento de como o envelhecimento pode estar relacionado com a saúde, é preciso observar os fatores biológicos, físicos, psicológicos, econômicos e sociais ${ }^{6}$. Fatores influenciam na qualidade de vida do idoso, como o lazer, o convívio familiar, relações no trabalho, atividade física e uso de medicamentos ${ }^{7,8}$.

0 envelhecimento ativo é necessário para minimizar com que ocorra possíveis limitações na capacidade funcional da população idosa, por meio da conservação da autonomia e independência, causando o bem-estar físico ${ }^{9,10}$. Desta forma, a autoestima e qualidade de vida são vistas como relativas e multidimensionais, de acordo com os aspectos positivos e negativos da vida e é determinada através da percepção e atitude na vida do indivíduo ${ }^{11}$.

As políticas destinadas aos idosos devem levar em consideração a capacidade funcional do idoso, além de incentivar a autonomia, a participação, o cuidado e a autossatisfação, abrangendo a prevenção e o cuidado integral à saúde do idoso, tendo como base a qualidade de vida e o envelhecimento ativo ${ }^{12}$. Dentro deste contexto, foram criados mecanismos para a organização e implantação de redes estaduais de assistência à pessoa idosa, incluindo o Centro de Referência do Idoso $(\mathrm{CRI})^{13}$.

Traçar um perfil desses idosos é um desfecho importante, visto que pacientes idosos geralmente sofrem de doenças crônicas e aumento da incapacidade e muitas das abordagens terapêuticas nessa faixa etária visam aliviar o sofrimento e melhorar o estado funcional dos pacientes. Avaliar a qualidade de vida dos indivíduos que frequentam um serviço dedicado exclusivamente a essa população pode então fornecer informações importantes para o desenvolvimento de políticas de saúde que atendam às reais necessidades de saúde dos idosos.
O objetivo desse estudo foi descrever o perfil sociodemográfico, da qualidade de vida, do nível de atividade física e morbidades referidas de idosos de um CRI no interior do estado de São Paulo, e compará-los aos idosos do Brasil.

\section{METODOLOGIA}

Trata-se um estudo comparativo e transversal, que foi aprovado pelo Comitê de Ética e Pesquisa (CEP) da Universidade do Oeste Paulista - UNOESTE (CAAE no 57330816.8.0000.5515 / protocolo 3301). Os idosos foram comunicados sobre os procedimentos da pesquisa, que foram realizados após a concordância e assinatura do Termo de Consentimento Livre e Esclarecido em consonância com a Resolução 466/2012 da CONEP.

A pesquisa foi realizada em um centro de referência do idoso (CRI) no interior do Estado de São Paulo. Esse centro caracteriza-se como sendo um local para prática de exercícios físicos regulares diários.

A amostra foi composta de 100 idosos frequentadores do CRI, com idades superiores a 60 anos dos sexos masculino e feminino. Foram incluídos idosos com avaliação cognitiva normal atestada por meio do questionário do Mini Exame do Estado Mental (MEEM) ${ }^{14}$, e que realizavam exercícios físicos no mínimo duas vezes por semana. Foram excluídos idosos que não conseguiram responder aos questionamentos durante as entrevistas.

A avaliação do nível sociodemográfico foi realizada por meio de questionário elaborado pelas pesquisadoras sobre os dados pessoais, escolaridade, profissão, renda salarial, composição familiar e moradia.

Para a avaliação da qualidade de vida foi aplicado o questionário WHOQOL-OLD ${ }^{15}$, que avalia funcionamento sensório, autonomia, atividades passadas, presentes e futuras, participação social, morte e medo de morrer e intimidade. $\mathrm{O}$ escore menor que 16 representa baixa qualidade de vida e um escore maior que 20 representa alta qualidade de vida.

Para avaliar o nível de atividade física dos participantes foi aplicado o questionário de Baecke $^{16}$, que avalia o nível da atividade física ocupacional, exercícios físicos no lazer, atividades de lazer e locomoção, nas categorias fisicamente inativo, moderadamente ativo A, moderadamente ativo $B$ e ativo. 
Por fim, investigou-se por meio do inquérito de morbidades referidas ${ }^{17}$ as doenças diagnosticadas nos últimos 10 anos dos seguintes eixos: doenças metabólico-endócrinas, cardiovasculares e osteomusculares.

Par análise dos dados foi utilizado o programa estatístico GraphPad Prism versão 5.0. A normalidade da distribuição dos dados foi avaliada por meio do teste de Shapiro-Wilk, e a descrição dos resultados foi realizada como porcentagens e média \pm desvio padrão (DP). Para análise intergrupos dos dados em porcentagem foi utilizado o teste de Qui-quadrado. $\mathrm{Na}$ comparação entre homens e mulheres foi utilizado o teste de Mann-Whitney. Na análise do nível de atividade física foi realizado uma divisão do escore total por quartil para classificar a amostra em fisicamente inativo ( $\leq$ P25), moderadamente ativo A (> P25 e $\leq$ P50), moderadamente ativo $\mathrm{B}(>\mathrm{P} 50 \mathrm{e} \leq \mathrm{P} 75)$ e ativo (>
P75). Para comparar os tipos de atividade física (lazer, locomoção e ocupacional) entre os quartis formados foi utilizado o teste de Kruskal-Wallis com pós teste de Dunn. O nível de significância utilizado foi de $p<0,05$.

\section{RESULTADOS}

A respeito dos aspectos sociodemográficos, foi observado que o gênero feminino foi predominante $(81 \% ; p=0,0004)$, com idade entre 70 e 74 anos e casados (46\%). Com relação à profissão, $82 \%$ eram aposentados e $47 \%$ dos idosos apresentam ensino fundamental. Dos entrevistados, $85 \%$ relataram ter moradia própria e renda entre um a dois salários mínimos, $48 \%$ possuíam carro próprio e $87 \%$ possuíam plano de saúde.

As características dos idosos do CRI assim como os idosos do Brasil (dados do IBGE) estão expressas na Tabela 1.

Tabela 1. Dados sociodemográficos dos idosos do Brasil (IBGE) e do CRI. Valores expressos em porcentagem.

\begin{tabular}{|c|c|c|c|c|}
\hline & & IBGE (\%) & CRI (\%) & $\mathbf{p}$ \\
\hline \multirow{2}{*}{ Sexo } & Feminino & 57 & 81 & \multirow{2}{*}{$0,0004^{*}$} \\
\hline & Masculino & 43 & 19 & \\
\hline \multirow{3}{*}{ Escolaridade } & Ensino fundamental & 89 & 47 & \multirow{3}{*}{$<0,0001^{*}$} \\
\hline & Ensino médio & 7 & 28 & \\
\hline & Superior & 4 & 25 & \\
\hline \multirow{4}{*}{ Estado civil } & Solteiro & 48 & 12 & \multirow{4}{*}{$<0,0001^{*}$} \\
\hline & Casado & 40 & 46 & \\
\hline & Viúvo & 6 & 33 & \\
\hline & Separado & 5 & 9 & \\
\hline \multirow{4}{*}{ Moradia } & Própria & 88 & 85 & \multirow{4}{*}{0,2697} \\
\hline & Alugada & 11 & 7 & \\
\hline & Cedida & 6 & 8 & \\
\hline & Mora com parente & 3 & 0 & \\
\hline \multirow{5}{*}{ Renda } & Até 1 salário mínimo & 16 & 22 & \multirow{5}{*}{$<0,0001^{*}$} \\
\hline & 1 a 2 salários mínimos & 27 & 52 & \\
\hline & 2 a 5 salários mínimos & 30 & 21 & \\
\hline & Acima de 5 salários mínimos & 11 & 5 & \\
\hline & Não sabe & 16 & 0 & \\
\hline \multirow{2}{*}{ Profissão } & Aposentado & 64 & 82 & \multirow{2}{*}{$0,0068 *$} \\
\hline & Não aposentado & 36 & 18 & \\
\hline
\end{tabular}

A Figura 1 representa os valores dos domínios de qualidade de vida dos idosos avaliados entre os sexos. Pode ser observada Colloq Vitae 2019 jan-abr; 11(1): 17-23. DOI: 10.5747/cv.2019.v11.n1.v248 ISSN 1984-6436/C 2018 - Publicado pela Universidade do Oeste Paulista. Artigo Open Access sob uma licença CC BY-NC-ND (http://creativecommons.org/licenses/by-nc-nd/4.0/). diferença significativa $(p<0,05)$ no domínio "Morte e Morrer". Não foram observadas diferenças significativas nos outros domínios. 


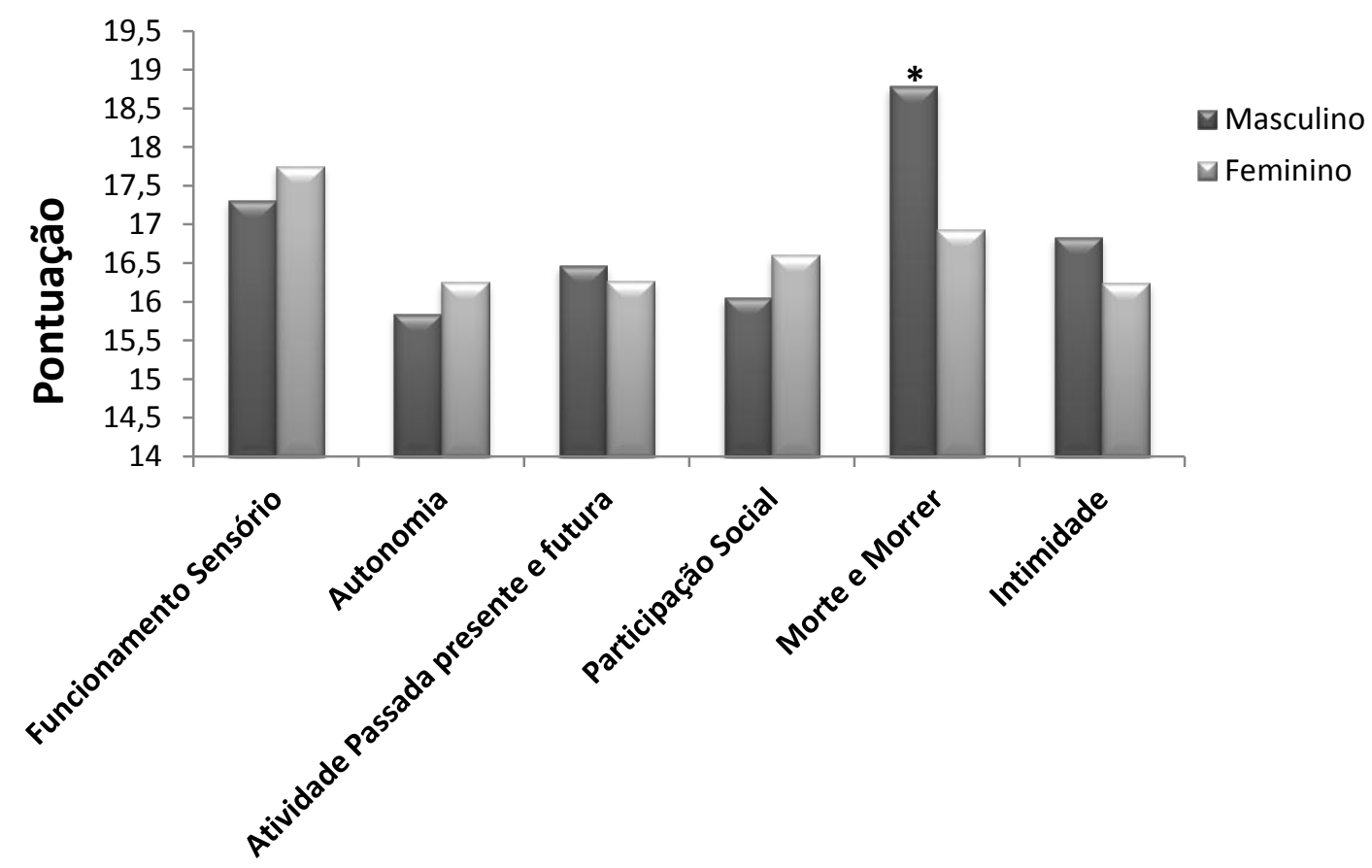

Figura 1. Valores de qualidade de vida de homens e mulheres do CRI. ${ }^{*} p<0,05$.

Quanto aos valores do MEEM, foram observados $26,73 \pm 2,72$ pontos no sexo masculino e $26,44 \pm 3,78$ pontos no sexo feminino. A respeito das morbidades referidas, na Tabela 2 é possível observar uma frequência maior em três doenças: dislipidemia (35\%), hipertensão arterial sistêmica $(58 \%)$ e artrite/artrose (37\%).

Tabela 2. Frequência de distúrbios metabólicos/endócrinos, cardiovasculares e osteomusculares dos idosos do CRI.

\begin{tabular}{llc}
\hline Doenças & Distúrbio & Frequência (\%) \\
\hline \multirow{3}{*}{ Metabólicas/endócrinas } & Dislipidemia & 35 \\
& Diabetes & 4 \\
& Hipertireoidismo & 0 \\
& Hipotireoidismo & 0 \\
\cline { 2 - 3 } & Hipertensão Arterial Sistêmica & 58 \\
Cardiovasculares & Arritmia & 4 \\
& Infarto & 3 \\
& Angina & 7 \\
\cline { 2 - 3 } & Osteoporose & 11 \\
& Artrite/artrose & 37 \\
Osteomusculares & Hérnia de disco & 10 \\
& Lombalgias & 5 \\
& Escoliose & 0 \\
& Todas & 1 \\
\hline
\end{tabular}


A Tabela 3 representa os resultados da avaliação do nível de atividade física. Os idosos foram considerados moderadamente ativos $(A=$ 38 e $B=37)$. Observou-se uma diferença significativa quando comparado fisicamente inativo e moderadamente ativo $B$; fisicamente inativo e ativo; moderadamente ativo $A$ e moderadamente ativo $B$; moderadamente ativo $A$ e ativo; e moderadamente ativo $B$ e ativo.

Tabela 3. Distribuição do nível de atividade física segundo as categorias de atividade física. Valores expressos em média e desvio padrão.

\begin{tabular}{lcccccc}
\hline & $\begin{array}{c}\text { Idosos } \\
(\mathrm{n}=100)\end{array}$ & $\begin{array}{c}\text { Fisicamente } \\
\text { inativo } \\
(\mathrm{N}=12)\end{array}$ & $\begin{array}{c}\text { Moderadamente } \\
\text { ativo } A \\
(\mathrm{~N}=38)\end{array}$ & $\begin{array}{c}\text { Moderadamente } \\
\text { ativo } B \\
(\mathrm{~N}=37)\end{array}$ & $\begin{array}{c}\text { Ativo } \\
(\mathrm{N}=13)\end{array}$ & $\mathbf{p}$ \\
\hline EFL & $5,41 \pm 0,53$ & $5,2 \pm 0,93$ & $5,47 \pm 0,41$ & $5,47 \pm 0,42$ & $5,29 \pm 0,64$ & 0,5794 \\
ALL & $2,47 \pm 0,75$ & $1,58 \pm 0,40$ & $2,16 \pm 0,51$ & $2,65 \pm 0,43$ & $3,70 \pm 0,49$ & $<0,0001^{*}$ \\
AFO & $3,57 \pm 0,52$ & $3,28 \pm 0,42$ & $3,40 \pm 0,47$ & $3,72 \pm 0,46$ & $3,91 \pm 0,63$ & $0,0005^{*}$ \\
Total & $6,02 \pm 0,85$ & $4,75 \pm 0,35$ & $5,52 \pm 0,29$ & $6,41 \pm 0,27$ & $7,51 \pm 0,37$ & $<0,0001^{*}$ \\
\hline
\end{tabular}

Legenda: EFL: exercício físico no lazer; ALL: atividade física de lazer e locomoção; AFO: atividade física ocupacional;

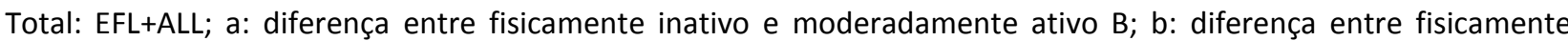
inativo e ativo; c: diferença entre moderadamente ativo A e moderadamente ativo B; d: diferença entre moderadamente ativo $\mathrm{A}$ e ativo; e: diferença entre moderadamente ativo $\mathrm{B}$ e ativo.

\section{DISCUSSÃO}

Os principais achados desse estudo mostraram que esses idosos são ativos e com uma qualidade de vida satisfatória. Os aspectos mais prevalentes sociodemográficos evidenciaram que os idosos avaliados do CRI caracterizava-se de uma população com estado civil casado, com renda de uma a dois salários mínimos e boa escolaridade quando comparados aos dados do IBGE.

Segundo a Organização Mundial da Saúde (OMS), idosos que possuem casa própria e que residem em ambientes mais seguros estão sujeitos a serem mais independentes, o que resulta em uma melhor qualidade de vida ${ }^{18}$.

Foi observado na presente pesquisa, que a renda dos idosos do CRI é menor quando comparados à população brasileira ${ }^{19}$. Quanto à escolaridade, a amostra apresentou um percentual maior em nível superior quando comparados ao Brasil, já no ensino fundamental se igualam ${ }^{19}$. Os achados de escolaridade e renda, por serem inversamente proporcionais, equilibram o desfecho na qualidade de vida dos idosos, pois outros estudos brasileiros apontaram que quanto menor a renda e a escolaridade, pior é a qualidade de vida ${ }^{20}$.
A frequência do gênero dos idosos avaliados foi semelhante aos dados do IBGE, onde se observaram uma maioria de idosos do sexo feminino. A expectativa de vida nas mulheres é mais longa do que os homens ${ }^{21}$. Explica-se tal fato de que as mulheres procuram meios de frequentar espaços sociais, atendimento médico e grupos de exercício físico, em comparação aos homens.

Em relação ao estado civil, da população estudada foram casados (46\%) seguindo de viúvos (33\%), no qual esses achados corroboram com outros estudos brasileiros ${ }^{22}$, apresentando prevalência de casados (45\%), e viúvos (43\%), e assim apresentam uma qualidade de vida satisfatória no geral, pois idosos com seus cônjuges têm melhor qualidade de vida quando comparados com idosos solteiros ou viúvos ${ }^{20}$.

As morbidades mais prevalentes foram dislipidemia, hipertensão arterial sistêmica e artrite/artrose, com destaque para a hipertensão arterial sistêmica (58\%), valores semelhantes aos do IBGE $(60,6 \%)^{22}$. Com isso observamos uma prevalência maior brasileira comparado a população estudada. Estudos analisaram que a implantação de Estratégia da Saúde da Família (ESF) pode ser uma política importante, pois 
proporciona educação em saúde como medida de prevenção os fatores de risco ${ }^{24}$.

A qualidade de vida dos idosos no domínio "morte e morrer" foi diferente entre os sexos, de forma em que as mulheres apresentaram maior medo de morrer do que os homens. Embora a expectativa de vida nas mulheres seja maior do que nos homens, elas apresentam etapas de incapacidades mais longas do que no sexo masculino, o que gera uma diferença na qualidade de vida, baseado no gênero ${ }^{18}$.

Observou-se que ambos os sexos tiveram uma boa participação social em grupos. Os exercícios físicos realizados em grupos promovem aos idosos uma troca de experiência, acolhimento e vínculos de amizade; além disso o idoso é capaz de socializar-se, sentir-se independente e minimizar sentimentos de solidão ${ }^{20}$.

O município de Presidente Prudente é considerado pelo Índice de Desenvolvimento Humano Municipal (IDHM), muito alto (IDHM entre 0,800 a 1), que pode ter influenciado positivamente na qualidade de vida dos idosos. A

\section{REFERÊNCIAS}

1. Vasconcelos AMN, Gomes MMF. Transição demográfica: a experiência brasileira. Epidemiol Serv Saúde. 2012;21(4):539-548.

DOI:

http://dx.doi.org/10.5123/S1679-

49742012000400003.

2. Azambuja MIR, Achutti AC, Reis RA, Siva JO, Fisher PD, Rosa RS et al. Saúde urbana, ambiente e desigualdades. Rev Bras Med Familía Comunid. 2011;6(19):110-115. DOI: https://doi.org/10.5712/rbmfc6(19)151.

3. Veras R. Envelhecimento populacional e as informações de saúde do PNAD: demandas e desafios contemporâneos. Introdução. Cad Saúde Púb. 2007;23(10):2463-6. DOI:

http://dx.doi.org/10.1590/s0102$\underline{311 \times 2007001000020}$.

4. Silva LWS, Santos RG, Squarcini CFR, Souza AL, Azevedo MP, Barbosa FNM. Perfil do estilo de vida e autoestima da pessoa idosa. Perspectivas de um programa de treinamento físico. Rev Temát Kair Geront. 2011;14(3):145-166.

5. Deponti RN, Acosta MAF. Compreensão dos idosos sobre os fatores que influenciam no envelhecimento contribuição para o IDHM do município, foi à longevidade apresentando índice de $0,858^{26}$.

Como limitações do estudo, uma pequena quantidade de pesquisas encontradas no campo da qualidade de vida e nível de atividade física de idosos brasileiros dificultou a discussão dos resultados, visto que esses dados são complexos por não serem comprovados estatisticamente. Por isso, sugerimos que esse tipo de pesquisa seja aprofundada em outros estudos.

O perfil sociodemográfico e a qualidade de vida dos idosos frequentadores de um centro de referência para idosos se igualou com a média brasileira e a superou em alguns perfis, o que nos faz relacionar com nível de atividade física dessa população e as políticas públicas direcionadas à terceira idade.

\section{CONFLITO DE INTERESSE}

Os autores declaram não haver qualquer potencial conflito de interesse que possa interferir na imparcialidade deste trabalho científico.

saudável. Estud Interdiscip Envelhec. 2010;15(1):3150.

6. The WHOQOL Group. The World Health Organization Quality of Life Assessment (WHOQOL): position paper from the World Health Organization. Soc Sci Med. 1995;41(10):1403-9. DOI: https://doi.org/10.1016/0277-9536(95)00112-K

7. Ferreira EEDBA. Gestão de custos com medicamentos, hidroginástica e qualidade de vida em pessoas idosas de Santarém. [dissertação]. Coimbra: Universidade de Coimbra, Faculdade de Ciências do Desporto e Educação Física, 2011.

8. Silva RS, Silva I, Silva RA, Souza L, Tomasi E. Atividade física e qualidade de vida. Ciênc Saúde Colet. 2010;15(1):115-20. DOI: http://dx.doi.org/10.1590/S141381232010000100017.

9. Brasil. Ministério da Saúde. Portaria no 2.528, de 01 de outubro 2006. Aprova a Política Nacional da Pessoa Idosa. 2006.

10. Néri, AL. Idosos no Brasil: vivências, desafios e expectativas na terceira idade. São Paulo: Fundação Perseu Abramo; 2007. Cap 1. 
11. The Whoqol Group. The world health organization quality of life assessment: position paper from the world health organization. Soc Sci Med. 1995; 41(10):1403-1409. DOI: https://doi.org/10.1016/0277-9536(95)00112-K

12. Miranda LCV, Soares SM, Silva PAB. Qualidade de vida e fatores associados em idosos de um Centro de Referência à Pessoa Idosa. Rev Ciên Saúde Col. 2016;21(11):3533-3544. DOI: http://dx.doi.org/10.1590/1413812320152111.21352015.

13. Brasil. Ministério da Saúde. Portaria no 702, de 12 de abril de 2002. Criação de mecanismos para a organização e implantação de Redes Estaduais de Assistência à Saúde do Idoso. 2002.

14. Brucki SMD, Nitrini R, Caramelli $P$, Bertolucci PHF, Okamoto IH. Sugestões para o uso do mini-exame do estado mental no Brasil. Arq Neuro-Psiquiatr. 2003; 61(3B):777-781.

DOI:

http://dx.doi.org/10.1590/S0004-

282X2003000500014.

15. Fleck MPA, Chachamovich E, Trentini CM. WHOQOL-OLD Project: method and focus group results in Brazil. Rev Saúde Púb. 2003;37(6):793-9. DOI: $\quad$ http://dx.doi.org/10.1590/S003489102003000600016 .

16. Florindo $A A$, Latorre MRDO. Validação e reprodutibilidade do questionário de Baecke de avaliação da atividade física habitual em homens adultos. Rev Bras Med Esporte. 2003;9(3):121-128.

19. Dawalibi NW, Goulart RMM, Prearo LC. Fatores relacionado à qualidade de vida de idosos em programa para a terceira idade. Rev Ciên Saúde Col. 2014;19(8):3505-3512.

DOI:

http://dx.doi.org/10.1590/1413-

81232014198.21242013.

18. Alexandre TS, Cordeiro RC, Ramos LR. Factors associated to quality of life in active elderly. Rev Saúde Pub. 2009;43(4):613-21. DOI: http://dx.doi.org/10.1590/S0034$\underline{89102009005000030 .}$.

19. Brasil. Instituto Brasileiro de Geografia e Estatística (IBGE). Síntese de indicadores sociais. 2016. Acesso em: 20 mai 2018. Disponível em https://www.ibge.gov.br/estatisticas- novoportal/sociais/educacao/9221-sintese-deindicadores-sociais.html?=\&t=downloads.

20. Varela FRA, Ciconelli RM, Campolina AG, Soarez PC. Quality of life evaluation of frail elderly in Campinas. Rev Assoc Med Bras. 2015;61(5):423-430. DOI: http://dx.doi.org/10.1590/1806-9282.61.05.423.

21. Leite MT, Hildebrandt LM, Kirchner RM, Winck MT, Silva LAA, Franco GP. Cognitive and health conditions in elderly participants in recreation and leisure activities centers. Rev Gáucha Enferm. 2012;33(4):6471. DOI: $\quad$ http://dx.doi.org/10.1590/S198314472012000400008 .

22. Guyatt GH, Eagle DJ, Sackett B, Willan A, Griffith L, Mcllroy $W$, et al. Measuring quality of life in the frail elderly. J Clin Epidemiol. 1993;46(12):1433-44. DOI: https://doi.org/10.1016/0895-4356(93)90143-O

23. Brasil. Ministério da Saúde. Datasus. Sistemas de informação sobre morbidades e mortalidades: indicadores e dados básicos. 2016. Acesso em: 20 mai 2018. Disponível em http://www2.datasus.gov.br/DATASUS/index.php?are $\mathrm{a}=0205$.

24. Cotta RMM, Batista KCS, Reis RS, Souza GA, Dias G, Castro FAF et al. Perfil sociossanitário e estilo de vida de hipertensos e/ou diabéticos, usuários do Programa de Saúde da Família no município de Teixeiras, MG. Ciên Saúde Col. 2009;14(4):1251-60. DOI: http://dx.doi.org/10.1590/S141381232009000400031.

25. Brasil. Atlas do desenvolvimento humano no Brasil. 2013. Acesso em: 20 mai 2018. Disponível em http://www.atlasbrasil.org.br/2013/pt/perfil m/presi dente-prudente

Recebido para publicação em 16/08/2018

Revisado em 24/08/2018

Aceito em 13/09/2018 